\title{
Campylobacter jejuni dsb gene expression is regulated by iron in a Fur-dependent manner and by a translational coupling mechanism
}

\author{
Anna D Grabowska ${ }^{1,2}$, Michał P Wandel ${ }^{1,3}$, Anna M Łasica', Monika Nesteruk ${ }^{1,4}$, Paula Roszczenko ${ }^{1}$, \\ Agnieszka Wyszyńska', Renata Godlewska ${ }^{1}$ and Elzbieta K Jagusztyn-Krynicka ${ }^{1 *}$
}

\begin{abstract}
Background: Many bacterial extracytoplasmic proteins are stabilized by intramolecular disulfide bridges that are formed post-translationally between their cysteine residues. This protein modification plays an important role in bacterial pathogenesis, and is facilitated by the Dsb (disulfide bond) family of the redox proteins. These proteins function in two parallel pathways in the periplasmic space: an oxidation pathway and an isomerization pathway. The Dsb oxidative pathway in Campylobacter jejuni is more complex than the one in the laboratory E. coli K-12 strain.

Results: In the C. jejuni 81-176 genome, the dsb genes of the oxidative pathway are arranged in three transcriptional units: $d s b A 2-d s b B-a s t A, d s b A 1$ and $d b a-d s b l$. Their transcription responds to an environmental stimulus - iron availability - and is regulated in a Fur-dependent manner. Fur involvement in dsb gene regulation was proven by a reporter gene study in a C. jejuni wild type strain and its isogenic fur mutant. An electrophoretic mobility shift assay (EMSA) confirmed that analyzed genes are members of the Fur regulon but each of them is regulated by a disparate mechanism, and both the iron-free and the iron-complexed Fur are able to bind in vitro to the C. jejuni promoter regions. This study led to identification of a new iron- and Fur-regulated promoter that drives dsbA1 gene expression in an indirect way. Moreover, the present work documents that synthesis of Dsbl oxidoreductase is controlled by the mechanism of translational coupling. The importance of a secondary dba-dsbl mRNA structure for $d s b /$ mRNA translation was verified by estimating individual $d s b /$ gene expression from its own promoter.
\end{abstract}

Conclusions: The present work shows that iron concentration is a significant factor in dsb gene transcription. These results support the concept that iron concentration - also through its influence on $d s b$ gene expression might control the abundance of extracytoplasmic proteins during different stages of infection. Our work further shows that synthesis of the Dsbl membrane oxidoreductase is controlled by a translational coupling mechanism. The $d b a$ expression is not only essential for the translation of the downstream $d s b /$ gene, but also Dba protein that is produced might regulate the activity and/or stability of Dsbl.

\section{Background}

Campylobacter jejuni is a human pathogen and the leading cause of acute bacterial gastroenteritis. As a commensal organism for many warm-blooded animals, especially in the gastrointestinal tract of poultry, $C$ jejuni is also isolated from a wide variety of watery environmental

\footnotetext{
* Correspondence: kjkryn@biol.uw.edu.pl

'Department of Bacterial Genetics, Institute of Microbiology, University of

Warsaw, Miecznikowa 1, 02-096, Warsaw, Poland

Full list of author information is available at the end of the article
}

sources $[1,2]$. Thus, the ability of $C$. jejuni to sense and respond to diverse environmental stimuli and to adapt gene expression to changes in external conditions is crucial for its pathogenesis, commensalism and survival outside the host organism.

Recent experiments have revealed many changes in the C. jejuni transcriptome and proteome that are driven by environmental stimuli. These include temperature, oxygen tension, iron concentration, sodium deoxycholate concentration and $\mathrm{pH}$ of the culture medium [3-7].

\section{Biomed Central}


C. jejuni's phase of life - planktonic vs biofilm - also shows a great difference in the microorganism's protein profile $[8,9]$. Campylobacter gene expression is coupled to environmental cues mostly by two-component signal transduction systems (TCSTS) [10-14]. The activity and the amount of a specific protein can also be affected by posttranslational modifications such as glycosylation, proteolysis and disulfide bond formation. That latter protein modification, which very often influences the tertiary and quaternary structure of virulence determinants, plays an important role in bacterial pathogenesis $[15,16]$. In Gram-negative bacteria disulfide bond formation is facilitated by the Dsb (disulfide bond) family of redox proteins, which function in the periplasmic space under oxidizing conditions. In E. coli the disulfide bridge formation system operates in two partially coinciding metabolic pathways: the oxidation (DsbA and DsbB) pathway and the isomerization/reduction (DsbC and DsbD) pathway. The oxidation pathway is responsible for the formation of disulfide bonds in newly synthesized proteins, just after they cross the cytoplasmic membrane. This process occurs in a rather non-selective way. The isomerization/ reduction pathway rearranges improperly introduced disulfides $[15,16]$.

The sequencing of more and more bacterial genomes has revealed that the process of disulfide bond formation in bacteria is extremely diverse, and it has become obvious that $E$. coli Dsb system cannot be considered a paradigm for Dsb activity $[16,17]$. The Dsb oxidative pathway of $C$. jejuni is much more complex than the oxidative pathway of the laboratory E. coli K-12. Depending on the strain, it is catalyzed by three or four enzymes two localized in the inner membrane (DsbB and DsbI) and one or two in the periplasm (DsbA1 and DsbA2). DsbA1 and DsbA2 possess classic signal sequences, which potentially ensure their transport through the cytoplasmic membrane into the periplasm. They are both directly responsible for disulfide bond formation. DsbB and DsbI, orthologues of E. coli DsbB, are potentially involved in DsbA1/DsbA2 re-oxidation [18]. C. jejuni genes of the Dsb oxidation pathway are organized in two clusters located at different chromosomal loci: dsbA2$d s b B-a s t A-d s b A 1$ and $d b a-d s b I$. AstA (arylsulfatase), encoded by the gene located in the first cluster, transfers arylsulfate groups between aromatic substrates in an adenosine 3'-phosphate-5'phosphosulfate (PAPS)-independent manner, at least in an E. coli strain [19-21], and is a substrate for the Dsb oxidative pathway. Based on specificity toward the donor aromatic substrate, arylsulfatases are classified as PAPS-dependent or PAPS-independent enzymes. The mode of $C$. jejuni AstA action remains uncharacterized. The $d b a$ gene encodes a potential protein of unknown function. Except for $d s b A 2, C$. jejuni $d s b$ genes are highly conserved within the species. Only $d s b A 2$ is variable among strains [15].

An active Dsb system is required for intestinal colonization by Campylobacter, as shown in a chicken infection model. Additionally, C. jejuni strain 81-176 with a mutated $d s b B$ or $d s b I$ gene showed reduced invasion/ intracellular survival ability in T84 cells. These data indicate that some targets of the Dsb system are involved in crucial processes of Campylobacter pathogenicity and commensalism [22].

The goal of this work was to analyze $C$. jejuni $d s b$ oxidative gene expression by characterizing its transcriptional units, and identify control mechanisms and environmental regulatory factors that facilitate the pathogen's adaptation to varying living conditions. We show that the $d s b$ genes are arranged in three operons in the genome, and that expression of those operons responds to an environmental stimulus - iron availability. Although transcription of $d s b B$ and $d s b I$ are both altered by iron concentration with Fur protein engagement, they are regulated differently. Thus, by changing Dsb protein abundance, the pathogen can regulate the amounts of many extracytoplasmic virulence factors that are substrates of the Dsb system, depending on the environmental conditions. Additionally, results show that synthesis of DsbI oxidoreductase is strongly controlled by the mechanism of translational coupling.

\section{Methods}

Bacterial strains, plasmids, media and growth conditions Bacterial strains and plasmids used in this study are listed in Table 1. C. jejuni strain 81-176 [23], and 480 [24] were grown under microaerobic conditions at $37^{\circ} \mathrm{C}$ in Mueller Hinton (MH) broth, on $\mathrm{MH}$ agar or Blood Agar Base No. 2 (BA) containing $5 \%$ horse blood. E. coli strains were grown at $37^{\circ} \mathrm{C}$ in Luria Bertani (LB) broth or on LB agar. When appropriate, the media were supplemented with antibiotics [Campylobacter Selective Supplement (Oxoid), ampicillin $(100 \mu \mathrm{g} / \mathrm{ml})$, chloramphenicol $(15 \mu \mathrm{g} / \mathrm{ml})$, kanamycin $(30 \mu \mathrm{g} / \mathrm{ml})$ or tetracycline $(10 \mu \mathrm{g} / \mathrm{ml})]$, iron sulfate $\mathrm{Fe}_{2}\left(\mathrm{SO}_{4}\right)_{3}(40 \mu \mathrm{M}$ - final concentration in all experimets) or the iron chelator deferoxamine mesylate $(20 \mu \mathrm{M}$ - final concentration in all experimets), X-Gal (13 mg/ml) and/or IPTG (3 mg/ml) in DMF (dimethyl-formamide).

As previously reported [6], growth of the C. jejuni NCTC 11168 was slower in the presence of deferoxamine mesylate (iron-restricted conditions) than in the presence of iron sulfate (iron-rich conditions). This was also observed for C. jejuni 81-176, so in iron-restricted conditions the strain was cultivated 5-7 hours longer than in iron-sufficient or iron-rich conditions, till the culture reached $\mathrm{OD}_{600}$ of about 0.4-0.6. We also noted that growth of the fur::cat mutated $C$. jejuni strains was 
Table 1 Bacterial strains and plasmids used in this study

\begin{tabular}{|c|c|c|}
\hline Strain/plasmid & Genotype or relevant characteristics & Origin \\
\hline \multicolumn{3}{|l|}{ C. jejuni strains } \\
\hline $81-176$ & parental strain; pVir, pTet $\left(\operatorname{Tet}^{R}\right)$ & G. Perez - Perez * \\
\hline AG1 & 81-176 dba::aphA-3 & This study \\
\hline AL1 & 81-176 dsbl::cat & This study \\
\hline AG6 & 81-176 $\Delta d b a-d s b l:: c a t$ & This study \\
\hline AG11 & 81-176 fur::cat & This study \\
\hline 480 & parental strain & J. van Putten ** \\
\hline AL4 & 480 dsbl::cat & This study \\
\hline AG15 & 480 fur::cat & This study \\
\hline \multicolumn{3}{|l|}{ E. colistrains } \\
\hline $\mathrm{DH} 5 \alpha$ & $\mathrm{F}^{-}$Ф80d lacZ $\triangle \mathrm{M} 15 \Delta\left(\right.$ lacZYA-orgF)U169 deoR recAlendA1 hsdR17 $\left(\mathrm{r}_{\mathrm{k}}^{-} \mathrm{m}_{\mathrm{k}}^{+}\right)$phoA supE44 $\lambda^{-}$thi-1 gyrA96 relA1 & Gibco BRL \\
\hline TG1 & supE44 hsd 5 thi $\Delta\left(\right.$ lac- proAB) $F^{\prime}\left[\operatorname{traD} 36\right.$ proAB ${ }^{+}$lacl lacZDM15] & {$[26]$} \\
\hline S17-1 & $\begin{array}{l}\text { recA pro hsdR RP4-2-Tc::Mu-Km::Tn7 } \\
\text { Tmpr', Spc', Str' }^{r}\end{array}$ & {$[56]$} \\
\hline \multicolumn{3}{|c|}{ General cloning/Plasmid vectors } \\
\hline pGEM-T Easy & $A p^{r} ; \operatorname{Lac} Z \alpha$ & Promega \\
\hline pRY107 & $\mathrm{Km}^{\mathrm{r}}$; E. coli/C. jejuni shuttle vector & {$[27]$} \\
\hline pRY109 & $\mathrm{Cm}^{\text {r; }}$ E. coli/C. jejuni shuttle vector & {$[27]$} \\
\hline pRK2013 & $\mathrm{Km}^{\mathrm{r}}$; helper vector for E. coli/C. jejuni conjugation & {$[28]$} \\
\hline \multicolumn{3}{|c|}{$\begin{array}{l}\text { Plasmids for gene expression study } \\
\text { Cj stands for PCR-amplified C. jejuni 81-176 DNA fragment (PCR primers are given in brackets) } \\
\text { Cc stands for PCR-amplified C.coli 72Dz/92 DNA fragment (PCR primers are given in brackets) } \\
\text { cj stands for C. jejuni 81-176 gene }\end{array}$} \\
\hline pUWM471 & pMW10/1300 bp Cc (HOB - H4X) & [39] \\
\hline pUWM803 & pMW10/440 bp Cj (Cjj879B - Cjj880X) & This study \\
\hline pUWM792 & pMW10/1170 bp Cj (Cjj879B - Cjj881X) & This study \\
\hline pUWM795 & pMW10/1980 bp Cj (Cjj879B - Cjj882X) & This study \\
\hline pUWM832 & pMW10/690 bp Cj (Cjj880B - Cjj880X) & This study \\
\hline pUWM833 & pMW10/750 bp Cj (Cjj880B2 - Cjj881X) & This study \\
\hline pUWM834 & pMW10/900 bp Cj (Cjj881B - Cjj882X) & This study \\
\hline pUWM864 & pMW10/660 bp Cj (Cjj882B3 - Cjj883X2) & This study \\
\hline pUWM827 & pMW10/540 bp Cj (Cj19LX-2 - Cj18Bgl) & This study \\
\hline pUWM828 & pMW10/720 bp Cj (Cj19LX-2 - Cj17Bgl) & This study \\
\hline pUWM858 & pMW10/240 bp Cj (Cjj45B - Cjj44X) & This study \\
\hline \multicolumn{3}{|c|}{ Plasmids for mutagenesis } \\
\hline pAV80 & pBluescript II SK/cjfur::cat & {$[25]$} \\
\hline pUWM622 & pBluescript II KS/cjdba::aphA-3 & This study \\
\hline pUWM713 & pGEM-T Easy/cjdsbl::cat & This study \\
\hline pUWM867 & pGEM-T Easy/Acjdba-cjdsbl:::cat & This study \\
\hline \multicolumn{3}{|c|}{ Plasmids for translational coupling study } \\
\hline pUWM769 & pRY107/cjdba-cjdsbl operon & This study \\
\hline pUWM811 & pRY107/cjdba (M1R)-cjdsb/ operon & This study \\
\hline pUWM812 & pRY107/cjdba (L29stop)-cjdsbl operon & This study \\
\hline pUWM1072 & pBluescript II SK/promoter of cjdba-cjdsb/ operon & This study \\
\hline pUWM1100 & pBluescript II SKcjdsb/ with its own promoter & This study \\
\hline pUWM1103 & pRY107/cjdsb/ with its own promoter & This study \\
\hline \multicolumn{3}{|c|}{ Plasmid for recombinant protein synthesis and purification } \\
\hline pUWM657 & $\mathrm{pET} 28 \mathrm{a} / c j d s b /$ (1100 bp 5'-terminal fragment) & This study \\
\hline pUWM1098 & pET24d/cjfur (fur coding region) & This study \\
\hline
\end{tabular}

* New York University School of Medicine, USA.

** Utrecht University, The Netherlands. 
markedly slower in iron-rich conditions than that of the wild type strain, but it was not slower in iron-restricted conditions. A similar inhibitory effect of iron chelation on the growth of $C$. jejuni 11168 was previously reported by van Vliet $[6,25]$.

\section{General DNA procedures}

Standard procedures for plasmid DNA isolation and DNA analysis were carried out as described by Sambrook and Russel [26] or were performed according to the manufacturer's instructions (A\&A Biotechnology). Synthetic primers synthesis (sequences given in Table 2) and DNA sequencing were performed in the DNA Sequencing and Oligonucleotide Synthesis Laboratory at the Institute of Biochemistry and Biophysics, Polish Academy of Sciences.
All vectors containing transcriptional fusions of putative $d s b$ gene promoter regions with a promotorless $l a c Z$ gene were constructed using the pMW10 E. coli/C. jejuni shuttle vector. DNA fragments were amplified from C. jejuni 81-176 chromosomal DNA with appropriate pairs of primers (listed in Table 2). Next, PCR products were cloned in the pGEM-T Easy vector (Promega), excised by restriction enzymes and subsequently cloned into pMW10, forming transcriptional fusions with the downstream promoterless lac $Z$ reporter gene. Correct construction of the resulting shuttle plasmids was confirmed by restriction analysis and sequencing. All recombinant plasmids, as well as the empty pMW10, were introduced into C. jejuni 480 cells by electroporation.

Construction of a pUWM1072 plasmid containing $d s b I$ without $d b a$ under its native promoter was achieved by

Table 2 Oligonucleotides used in the present study

\begin{tabular}{|c|c|c|}
\hline Name & Sequence & Orientation//restriction site \\
\hline $\mathrm{HOB}$ & GTCTAGGATCCGCTTGATATCGCTGATTACT & Fwd//BamHI \\
\hline $\mathrm{H} 4 \mathrm{X}$ & ATCTGTCTAGAGCCAGCAGGAGCAATTACATCT & Rev//Xbal \\
\hline Cj16RS & GCAGTCGACTCAATGAAGGTAAGAGTAAG & Rev//Sall \\
\hline $\mathrm{Cj} 17 \mathrm{Bgl}$ & CCTAGATCTAGCCTGCTAAACACATTAGT & Rev//BgllI \\
\hline Cj17Nde & GTACATATGAACGAAATCAATAAAAC & Fwd//Ndel \\
\hline Cj17RBgl & TTCAGATCTCTAATGTGTTTAGCAGGC & Fwd//Bglll \\
\hline Cj17RM & TATGAATTCAGGAATACCTGTGCTAACAA & Rev//EcoRI \\
\hline Cj17LSal & GCTGTCGACTGATAAGAAAGAATATTG & Rev//Sall \\
\hline Cj17WDBam-low & GGATCCTGTGGGGAGTGCGATAG & Rev//BamHI \\
\hline Cj17WDBam-up & GGATCCACAGGTATTCCTCCTTATGTAG & Fwd//BamHI \\
\hline Cj18Bgl & CCTAGATCTGATAATCAGTATCAAGGCGA & Rev//BgllI \\
\hline Cj18L29 & CCAAGCTACCATTACCTAACCAAAAGCCAAAT & $\operatorname{Rev} / / \varnothing$ \\
\hline Cj18L29_C & ATTTGGCTTTTGGTTaGGTAATGGTAGCTTGG & Fwd $/ / \varnothing$ \\
\hline Cj18LM & TATGGATCCCAGGAGCACTATTAACAATA & Fwd//BamHI \\
\hline Cj18M1R & AAAGTTCAAGAAACTCCCTAGTATCTCCTTTG & $\operatorname{Rev} / / \varnothing$ \\
\hline Cj18M1Rc & CAAAGGAGATACTAgGGAGTTTCTTGAACTTT & Fwd//Ø \\
\hline Cj18Nde-Rev & ATACTGCAGCAAGAAACTCCATATGATCTCC & Rev//Pstl, Ndel \\
\hline Cj18RM & TGAGGATCCAAGCCAAGCTACCATTACCA & Rev//BamHI \\
\hline Cj19LX-2 & AGTTCTAGAAGTTGGACAGCTTGCTGATA & Fwd//Xbal \\
\hline Cjj43Eco & GCAGAATTCAAGCATAGCAGGATCTTTGG & Rev//EcoRI \\
\hline Cjj43mwL & CGTGGATCCCCGGGTAGGACTTATATTTAATC & Fwd//Smal, BamHI \\
\hline Cjj44X & AGTTCTAGACATTTAGCCCTTCCTTACAG & Rev//Xbal \\
\hline$C \mathrm{jj} 45 \mathrm{~B}$ & ATCGGATCCGATACTATGGAGTTTCTTGA & $\mathrm{Fwd} / / \mathrm{BamHI}$ \\
\hline Cjj45Dig & TCAAGAAACTCCATAGTATC & $\operatorname{Rev} / / \varnothing$ \\
\hline$C \mathrm{jj} 46$ & CATGTGAAATCAATAATATC & Fwd//Ø \\
\hline Cjj46mwR & ATACCCGGGGATCCAAAGTTACTGAAAGCTAC & Rev//Smal, BamHI \\
\hline Cj-RT & GCAGTCGACTAGGATCGATAGTAGCTGAA & Rev//Sall \\
\hline Cjj879B & ATCGGATCCCACAATCTAAAGGGTATTTC & Fwd//BamHI \\
\hline Cjj880 & СТTАТССАТАААATATAATG & Fwd//Ø \\
\hline Cjj880B & ATCGGATCCACCTAGATTATTCTACTTTG & Fwd//BamHI \\
\hline Cjj880B2 & ATCGGATCCTTTCCAGTAAAATTAGCAAG & Fwd//BamHI \\
\hline Cjj880X & AGTTCTAGACACATACAACAATAGATCTT & Rev//Xbal \\
\hline Cjj881B & ATCGGATCCAAAATGAAAGATAATTGCAG & $\mathrm{Fwd} / / \mathrm{BamHI}$ \\
\hline C jjj881X & AGTTCTAGAAAATGTGCTATACAAGTAAG & Rev//Xbal \\
\hline
\end{tabular}


Table 2 Oligonucleotides used in the present study (Continued)

\begin{tabular}{|c|c|c|}
\hline Cjj882 & AGGTGTAAGTCTTGGAGAGC & Fwd $/ / \varnothing$ \\
\hline Cjj882B & ATGGATCCGACTTAGCAAAACTCTTTGT & Fwd//BamHI \\
\hline Cjj882X & AGTTCTAGAAGAGTTTTGCTAAGTCTCAT & Rev//Xbal \\
\hline Cjj882B3 & ATCGGATCCATGATGATTATAGCAAATGC & Fwd//BamHI \\
\hline Cjj883X2 & AGTTCTAGAGCTAATGCAAAACTTGAATA & Rev//Xbal \\
\hline$C M-L$ & ATATCACGCAATTAACTTGG & $\operatorname{Rev} / / \varnothing$ \\
\hline$C M-R$ & GGATGAATTACAAGACTTGC & Fwd $/ / \varnothing$ \\
\hline DIG_dsbA1 & GCTAATGCAAAACTTGAATA & $\operatorname{Rev} / / \varnothing$ \\
\hline DIG_dsbA2X & CACATACAACAATAGATCTTG & $\operatorname{Rev} / / \varnothing$ \\
\hline DIG_chuF & СATATGAGAAATAATGCTTTC & Fwd $/ / \varnothing$ \\
\hline EMSAchuR & TTTGGGTGCAAATTTTACTC & $\operatorname{Rev} / / \varnothing$ \\
\hline Fur-L & TGAATTTTTATTGGTTTGATGC & Fwd $/ / \varnothing$ \\
\hline Fur-R & TCСТСАТСТTСАATGTTTGC & $\operatorname{Rev} / / \varnothing$ \\
\hline KAN-L & TATCACCTCAAATGGTTCGCTGGG & $\operatorname{Rev} / / \varnothing$ \\
\hline KAN-R & GGGGATCAAGCCTGATTGGGAGA & Fwd $/ / \varnothing$ \\
\hline KM-L1 & GAGAATATCACCGGAATTGA & Fwd $/ / \varnothing$ \\
\hline KM-R1 & СTTCATACTCTTCCGAGCAA & $\operatorname{Rev} / / \varnothing$ \\
\hline lacz & AGGTTACGTTGGTGTAGATG & $\operatorname{Rev} / / \varnothing$ \\
\hline lacZ1 & GGAATTCACTGGCCGTCGTT & Fwd $/ / \varnothing$ \\
\hline
\end{tabular}

Bold letters indicate C. jejuni 81-176 sequences; restriction recognition sites introduced for cloning purposes are underlined, complementary fragments of primers $\mathrm{C}_{\mathrm{jj}} 46 \mathrm{mwR}$ and $\mathrm{Cjj} 43 \mathrm{mwL}$ are marked with italics. Point mutated nucleotides in primers are marked with small letters. Orientation of the primers (Fwd states for forward/Rev - for reverse) refers to the orientation of particular C. jejuni gene studied. RT-Cj primer was designed on the basis of C. coli $72 \mathrm{Dz} / 92 \mathrm{ds} b / \mathrm{nucleotide}$ sequence (there are 2 nucleotide changes compared to the nucleotide sequence of its orthologue from C. jejuni 81-176).

PCR-amplification of the 520 bp chromosomal DNA fragments containing the $d b a-d s b I$ promoter sequences (primer pair Cj19LX-2 - Cj18Nde-Rev) and cloning it into pBluescript II SK (Stratagene), using XbaI/PstI restriction enzymes. Subsequently the $d s b I$ coding sequence (1792 bp) was PCR-amplified using the Cj17Nde - Cj16RS primer pair, cloned into pGEM-T Easy (Promega) and finally, using NdeI/SalI restriction enzymes, transferred into pUWM1072 in the native orientation, generating the plasmid pUWM1100. The whole insert (2316 bp) was then cloned into a shuttle E. coli/C. jejuni vector pRY107 [27] using SalI/XbaI restriction enzymes. The resulting, plasmid pUWM1103, whose correct construction was verified by sequencing, was used for complementation assays in C. jejuni $\Delta d b a-$ dsbI::cat mutant cells.

Point mutations were generated using a Quick-Change site-directed mutagenesis kit, following the supplier's recommendations (Stratagene). To construct a $d b a$ gene with point mutations, the pUWM456 plasmid, containing the $C$. jejuni $d b a-d s b I$ genes, was used as a template for PCR-mediated mutagenesis. Point mutations M1R and L29stop (replacing a Leu codon with amber stop codon) were introduced using the respective pairs of primers: Cj18M1R - Cj18M1Rc and Cj18L29 - Cj18L29c. The resulting plasmids were introduced into $E$. coli cells by transformation and presence of desired mutations was verified by DNA sequencing. DNA fragments containing the $C$. jejuni $d b a-d s b I$ operon (with or without a point mutation) were then digested and inserted into the pRY107 shuttle vector. The resulting plasmids were named pUWM769 (containing wt $d b a-d s b I$ ), pUWM811 (dba: M1R, wt $d s b I)$ and pUWM812 (dba: L29stop, wt $d s b I)$. These plasmids were subsequently introduced into C. jejuni 81-176 AL1 (dsbI::cat) and C. jejuni 81-176 AG6 ( $\Delta d b a-d s b I:: c a t)$ knock-out cells by conjugation [28].

\section{Construction of bacterial mutant strains}

To inactivate $d b a$ and $d s b I$ genes, three recombinant plasmids were constructed, based on pBluescript II KS (Stratagene) and pGEM-T Easy (Promega) vectors, which are suicide plasmids in C. jejuni cells. A. van Vliet kindly furnished the fourth suicide plasmid, pAV80, which was previously used for C. jejuni NCTC11168 fur inactivation [25]. Correct construction of all the plasmids was confirmed by restriction analysis and sequencing.

The plasmid for $C$. jejuni $d b a$ mutagenesis was generated by PCR-amplification of two C. jejuni 81-176 DNA fragments (600 bp and $580 \mathrm{bp}$ long) that contained $d b a$ gene fragments with their adjacent regions with primer pairs: Cj19LX-2 - Cj18RM and Cj18LM - Cj17RM. Next they were cloned in native orientation in pBluescript II KS (Statagene). Using BamHI restrictase, the kanamycin resistance cassette (the $1.4 \mathrm{~kb}$ aphA-3 gene excised from pBF14) was inserted between the cloned $d b a$ arms in the same transcriptional orientation, generating the suicide plasmid pUWM622. 
To obtain the construct for $C$. jejuni dsbI mutagenesis the $1.5 \mathrm{~kb}$ DNA fragment containing the $d s b I$ gene was PCR-amplified from the C. jejuni 81-176 chromosome using primer pair: $\mathrm{Cj} 17 \mathrm{LSal}$ - Cj17RBgl and was cloned into pGEM-T Easy (Promega). Subsequently, the internal 300 bp EcoRV-EcoRV region of $d s b I$ was replaced by a SmaI-digested chloramphenicol resistance cassette (the $0.8 \mathrm{~kb}$ cat gene excised from pRY109) [27] inserted in the same transcriptional orientation as the $d s b I$ gene, generating the suicide plasmid pUWM713.

To obtain the construct for $C$. jejuni $d b a-d s b I$ mutagenesis, the $410 \mathrm{bp}$ and $380 \mathrm{bp}$ DNA fragments, containing $d b a$ upstream and $d s b I$ downstream regions were PCR-amplified from the C. jejuni 81-176 chromosome using primer pairs: Cj19LX-2 - Cjj46mwR and Cjj43mwL - Cjj43Eco. These fragments were directly digested with BamHI restrictase, ligated in a native orientation and used as a template for a subsequent PCR reaction with the external primer pair: Cj19LX-2 - Cjj43Eco. This PCR product was cloned into pGEM-T Easy (Promega) and the chloramphenicol resistance cassette (the $0.8 \mathrm{~kb}$ cat gene excised from pRY109) was inserted in the same transcriptional orientation as $d b a-d s b I$ operon at the BamHI site between the C. jejuni DNA fragments, generating suicide plasmid pUWM866.

Gene versions inactivated by insertion of a resistance cassette were introduced into the $C$. jejuni $81-176$ or 480 chromosome by the allele exchange method as described by Wassenaar et al. [24]. Construction of the C. jejuni 480 fur::cat mutant was achieved by natural transformation using C. jejuni 81-176 fur::cat chromosomal DNA. It should be pointed out that C. jejuni 480 was previously described as incapable of accepting chromosomal DNA by natural transformation [24]. Such inconsistency of experimental data might be due to different chromosomal DNA used for natural transformation (C. jejuni 81116 vs C. jejuni 81-176). The mutant strains were obtained by two- or tri-parental mating experiments performed as described by Labigne-Roussel et al. [29] and Davis et al. [30]. The constructed mutants were named AG1 (C. jejuni 81-176 dba::aphA3), AL1 (C. jejuni 81-176 dsbI::cat), AL4 (C. jejuni 480 dsbI::cat), AG6 (C. jejuni 81-176 $\Delta$ dba-dsbI::cat), AG11 (C. jejuni 81-176 fur::cat), and AG15 (C. jejuni 480 fur:: cat). They demonstrated normal colony morphology and all but two had normal growth rates when cultured on BA plates. Only the C. jejuni 81-176 fur::cat and C. jejuni 480 fur::cat exhibited slower growth, an observation consistent with other studies on fur mutants [25]. Disruption of each gene as a result of double cross-over recombination was verified by PCR with appropriate pairs of primers flanking the insertion site (Table 2). The loss of DsbI synthesis in the constructed mutants was verified by Western blotting of whole-cell protein extracts against specific rabbit polyclonal anti-rDsbI antibodies.

\section{Protein manipulation, and $\beta$-galactosidase and arylsulfate sulfotransferase (AstA) assays}

Preparation of C. jejuni protein extracts, SDS-PAGE (sodium dodecyl sulfate polyacrylamide gel electrophoresis) and blotting procedures were performed by standard techniques [26].

To obtain recombinant $\mathrm{His}_{6}$-DsbI protein, the $1100 \mathrm{bp}$ DNA fragment containing the coding sequence for the predicted periplasmic DsbI C-region was PCR-amplified from the C. jejuni 81-176 chromosome using a primer pair: Cj17WDBam-up - Cj17WDBam-low. This fragment was cloned into the pGEM-T Easy vector and then, using BamHI restriction enzyme, into expression vector pET28a (Novagen) to generate plasmid pUWM657, whose correct construction was verified by restriction analysis and sequencing. Cytoplasm-located soluble fusion protein $\mathrm{His}_{6}$-DsbI purified from the E. coli Rosetta (DE3) LacI $^{\mathrm{q}}$ strain by affinity chromatography was used for rabbit immunization (Institute of Experimental and Clinical Medicine, Polish Academy of Science, Warsaw, Poland). The anti-His ${ }_{6}$-DsbI (anti-rDsbI) serum obtained was highly specific and recognized native DsbI, as verified by Western blot experiments carried out with protein extracts from $C$. jejuni wild type and a $d s b I$ mutant strain (data not shown).

To obtain recombinant Fur-His 6 protein, the DNA fragment containing the entire fur coding region was PCRamplified from the $C$. jejuni 81-176 chromosome with primer pair CjFurNcI - CjFurXhI, and then cloned, using NcoI/XhoI restriction enzymes, into pET24d (Novagen). This generated pUWM1098, carrying a fur-his 6 translational fusion. This plasmid was then transformed into E. coli BL21 (DE3) cells. Recombinant Fur-His ${ }_{6}$ protein was overproduced by addition of $1 \mathrm{mM}$ IPTG to the bacterial culture at exponential growth phase and purified under native conditions by affinity chromatography.

$\beta$-galactosidase activity assays in $C$. jejuni cell extracts were performed three times (each time three independent samples were taken for each strain), as described by Miller [31].

C. jejuni transformants grown overnight on BA medium were harvested and resuspended in LB medium to achieve comparable cell densities $\left(\mathrm{OD}_{600}\right.$ approx. 0.6). Fresh $\mathrm{MH}$ liquid medium ( $\mathrm{MH}$ supplemented with iron sulfate iron-rich conditions, $\mathrm{MH}$ itself - iron-sufficient and $\mathrm{MH}$ with iron chelated by addition of deferoxamine mesylate iron-restricted conditions) was inoculated with $C$. jejuni (1:10) and incubated overnight (15-22 $\mathrm{h}$ depending on the medium) till the culture reached $\mathrm{OD}_{600}$ of about 0.4-0.6. Since Wright et al. documented that $C$ jejuni exhibits a dynamic stationary phase, characterized by switches in 
motility, substrate utilization and metabolite production accompanied by concurrent changes in gene expression, exponential phase cultures were used in this experiment to eliminate any stationary phase-dependent physiological switching of gene expression levels [32].

Quantitative assays for AstA arylsulfatase activity were performed three times (each time three independent samples were taken for each strain), using the method described by Hendrixson et al. with one difference: the C. jejuni 81-176 strain was cultivated on $\mathrm{MH}$ liquid medium under high- or low-iron conditions [33] (approx. $17 \mathrm{~h}$ on $\mathrm{MH}$ medium under high iron condition and approx. $22 \mathrm{~h}$ on $\mathrm{MH}$ medium under low-iron condition). For each experiment, bacterial cultures of the same $\mathrm{OD}\left(\mathrm{OD}_{600} \sim 0.6-0.7\right)$ were used.

\section{RNA analysis}

Total RNAs were extracted from C. jejuni overnight BA culture using the standard TRIzol reagent according to the manufacturer's protocol (Invitrogen). RNA samples were treated with DNaseI to eliminate contaminating DNA and quantified by measurements of $\mathrm{OD}_{260}$, RNA was reverse transcribed using Superscript II enzyme (Invitrogen) and RT-primer (Table 2): Cj-RT complementary to the dsbIinternal fragment, or KM-R1, complementary to the kanamycin-resistance cassette. The RT primer was annealed stepwise before adding the reverse transcriptase. The enzyme was finally inactivated by incubation at $70^{\circ} \mathrm{C}$ for $15 \mathrm{~min}$. A control reaction without reverse transcriptase was used to determine RNA template purity from DNA. PCR reactions (with pairs of primers: Cj17Nde - Cj17RM or KM-L1 - KM-R1) performed on CDNA were carried out in the presence of $2 \mathrm{mM} \mathrm{MgCl}_{2}$ using the following protocol: initial denaturation at $94^{\circ} \mathrm{C}$ for $5 \mathrm{~min}$; then 30 cycles of: $30 \mathrm{~s}$ denaturation at $94^{\circ} \mathrm{C}, 30 \mathrm{~s}$ annealing at $50-60^{\circ} \mathrm{C}, 30-180 \mathrm{~s}$ elongation at $72^{\circ} \mathrm{C}$ and $10 \mathrm{~min}$ terminal elongation at $72^{\circ} \mathrm{C}$. Resulting PCR products were separated by electrophoresis in a $1.5 \%$ agarose gel.

RNA secondary structure was predicted by calculating a $100 \%$ consensus among different methods (Afold, PknotsRG, RNAfold, Contrafold, and RNAsubopt) run via the metaserver available at http://genesilico.pl/rnametaserver/.

\section{Gel mobility shift assay}

The promoter regions upstream of the $d b a-d s b I$ and dsbA2-dsbB-astA operons ( 180 bp and $\sim 330$ bp, respectively) and the $d s b A 1$ gene $(\sim 300 \mathrm{bp})$ as well as the CJJ81176_1600 - chuA intergenic spacer region ( 220 bp) which contains two Fur boxes (positive control) were PCR-amplified from C. jejuni 81-176 chromosomal DNA, using the following primer pairs: DIG_Cjj45 - Cjj46, DIG_dsbA2X - Cjj880, DIG_dsbA1 - Cjj882 and DIG_chuF - EMSAchuR. Primers: DIG_Cjj45, DIG_dsbA2X,
DIG_dsbA1 and DIG_chuF were digoxigenin labelled (Metabion). Approximately $28 \mathrm{fmol}$ of each DIG-labelled DNA fragment was incubated with 0, 333, 1000 or 3333 nM of purified Fur-His protein for $20 \mathrm{~min}$. at room temperature and subsequently for $5 \mathrm{~min}$. at $37^{\circ} \mathrm{C}$ in a $20 \mu \mathrm{l}$ volume of binding buffer routinely used for the Fur-binding assay (10 mM Tris- $\mathrm{HCl}[\mathrm{pH} 7.5], 1 \mathrm{mM} \mathrm{MgCl}_{2}, 0.5$ $\mathrm{mM}$ dithiothreitol, $50 \mathrm{mM} \mathrm{KCl}, 100 \mu \mathrm{M} \mathrm{MnCl}_{2}, 1 \mu \mathrm{g}$ poly (dI-dC), $50 \mu \mathrm{g}$ bovine serum albumin and $5 \%$ glycerol). In addition, $d s b A 2$ and $d s b A 1$ promoter regions were incubated with Fur-His protein in binding buffer without $\mathrm{Mn}^{2+}$. As negative controls each Dig-labelled DNA fragment was incubated with an unrelated protein (purified H. pylori HP0377- $\mathrm{His}_{6}$ ). Control reactions were performed using competitor DNA - unlabeled promoter DNA region. Samples were run on a $5 \%$ non-denaturing Tris-glycine polyacrylamide gel at $4^{\circ} \mathrm{C}$. Then DNA was transferred to nylon membranes (Roche) and UV crosslinked. Labelled DNA was detected with anti-DIG antibody using a standard DIG detection protocol (Roche).

\section{Results}

\section{In silico analysis of $C$. jejuni 81-176 $d s b$ gene clusters}

C. jejuni 81-176 dsbA2-dsbB-astA-dsbA1 genes (cjj81176_ 0880-0883) have the same orientation in the chromosome (Figure 1A) and are separated by short intergenic regions $11 \mathrm{bp}, 87 \mathrm{bp}$, and $85 \mathrm{bp}$, respectively. Thus, they potentially might be co-transcribed. In silico analysis of the $C$. jejuni dsbA2-dsbB-astA-dsbA1 cluster revealed the presence of a potential RBS as well as a complete promoter nucleotide sequence upstream of $d s b A 2$, located within the 627 bp intergenic $x e r D-d s b A 2$ region [34]. As this DNA fragment consists of $-35,-16$ and -10 regions (characteristic for the $\sigma^{70}$ binding sequence), it can be recognized by Campylobacter RNAP containing the main sigma factor. Directly upstream of $d s b B$ there is a potential additional RBS sequence but none of the promoter regions were found, suggesting $d s b A 2-d s b B$ co-transcription. Upstream of $a s t A$ and $d s b A 1$ there are putative RBS sequences and incomplete promoter nucleotide sequences, suggesting that $a s t A$ and $d s b A 1$ might be transcribed separately from $d s b A 2$ and $d s b B$.

C. jejuni 81-176 dba (cjj81176_0045c) and dsbI (cjj81176_0044c) have the same orientation in the chromosome (Figure 1B) and their coding sequences are separated by a short intergenic region of $11 \mathrm{bp}$. An initial RT-PCR experiment carried out on the total C. jejuni RNA documented $d b a-d s b I$ co-transcription in vitro and localization of their promoter within $493 \mathrm{bp}$ DNA upstream of the $d b a$ translation start codon [18].

\section{Transcriptional analysis of two $d s b$ gene clusters}

The lac $Z$ reporter gene system was used to determine the $d s b$ gene expression and regulation. Two sets of $d s b-l a c Z$ 


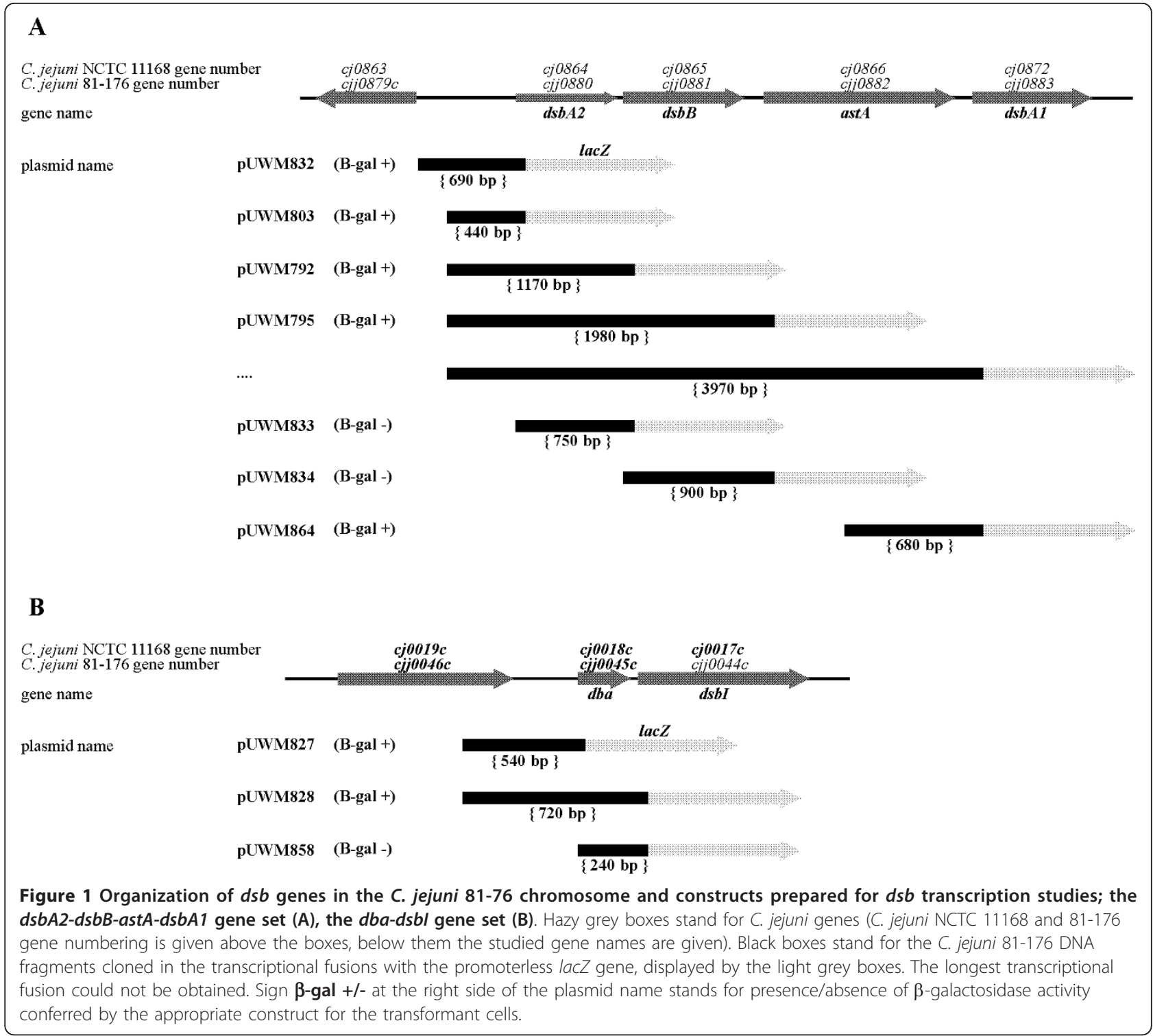

transcriptional fusions were designed based on a promotorless $l a c Z$ gene in the shuttle vector pMW10 [34]. The first one comprised of seven plasmids (pUWM792, pUWM795, pUWM803, pUWM832, pUWM833, pUWM834 and pUWM864) employed to study $d s b A 2 /$ $d s b B / a s t A / d s b A 1$ expression. The other consisted of three plasmids (pUMM827, pUWM828 and pUWM858) generated to analyze $d b a / d s b I$ expression. Details of the recombinant plasmid structures are shown in Figure 1. We successfully prepared all but one of the planned transcriptional fusions - we failed at constructing the longest fusion presented in Figure 1.

$\beta$-galactosidase assays indicated that the fusions present in pUWM833, pUWM834 and pUWM858 were not expressed in $C$. jejuni cells. This documented that the analyzed genes form two polycistronic operons
(dsbA2-dsbB-ast $A$ and $d b a-d s b I)$ and only $d s b A 1$ is independently transcribed. The level of $\beta$-galactosidase provided by the $d s b A 1$ promoter was approximately ten times higher than that conferred by the two other promoters that were analyzed (contained in PUWM803 and pUWM827). Thus, three promoters of various strengths and responsible for $C$. jejuni $d s b$ gene expression were identified: $\mathrm{P}_{d b a d s b I}, \mathrm{P}_{d s b A 2 d s b B a s t A}$ and $\mathrm{P}_{d s b A 1}$.

\section{Influence of environmental stimuli on $d s b$ gene expression}

We subsequently tested whether gene expression driven by $\mathrm{P}_{d s b A 2 d s b B a s t A}, \mathrm{P}_{d s b A I}$ and $\mathrm{P}_{d b a d s b I}(C$. jejuni 480 strains harbouring pUWM803, pUWM864 or pUWM827) responds to environmental stimuli. While there were no significant differences in $\beta$-galactosidase activity between 
cells grown at various temperatures $\left(37^{\circ} \mathrm{C}\right.$ and $\left.42^{\circ} \mathrm{C}\right)$ (Figure 2A) or between cells grown in solid and liquid medium ( $\mathrm{MH}$ broth and $\mathrm{MH}$ solidified by agar addition) (data not shown), transcription from each of the analyzed promoters was iron-regulated (Figure 2B). For cells grown in iron-restricted conditions, $\mathrm{P}_{d s b A 2 d s b B a s t A}$ activity was 10 times lower, $\mathrm{P}_{d s b A 1}$ activity was about $30 \%$ lower, and $\mathrm{P}_{d b a d s b I}$ activity was four times higher, compared to cells grown under iron-sufficient/iron-rich conditions.

Iron-regulated expression of many Gram-negative bacterial genes is mediated by the ferric uptake regulator (Fur) $[35,36]$. Classically, the Fur protein first binds to its co-repressor $\mathrm{Fe}^{2+}$, and then binds to the conserved DNA sequence (Fur-box) of the regulated promoter, repressing its transcription. However, transcriptomic analyses documented that apo-Fur (without complexed co-repressor) can also influence gene transcription in response to iron concentration [6,36-38].

We therefore decided to evaluate the regulatory function of the Fur protein on $d s b$ gene expression. For this purpose a C. jejuni 480 fur isogenic mutant was constructed. Then, recombinant plasmids containing $d s b$ promoter-lacZ fussions (pUWM803, pUWM864 and pUWM827) were introduced into the C. jejuni 480 fur:: cat mutant by electroporation. The results of $\beta$-galactosidase assays performed on the constructed strains proved Fur involvement in iron-dependent regulation of the three analyzed $d s b$ gene promoters (Figure $2 \mathrm{C}$ ). $\beta$ galactosidase activity conferred by the pUWM827 fusion increased under iron-sufficient/rich conditions in the fur mutant as compared to the wild-type strain, suggesting that inactivation of fur results in derepression of $\mathrm{P}_{d b a d s b I}$. In contrast, $\beta$-galactosidase activities of the pUWM803 and pUWM864 fusions increased under iron starvation in the fur mutant compared to the wild-type strain. This indicates that low level of iron leads to Fur-mediated repression of the $\mathrm{P}_{d s b A 2 d s b B a s t A}$ and $\mathrm{P}_{d s b A 1}$ promoters, since repression was abolished in the fur mutated strain. C. jejuni 480 strain containing pUWM471, which harbors cjaA gene promoter fused to a promotorless $l a c Z$ gene, was employed as a control in all experiments analyzing the influence of Fur and iron on $d s b$ gene expression. There were no significant differences in $\beta$ galactosidase activity between wild type cells harbouring pUWM471 grown at various iron concentrations as well as between wt and fur mutated cells containing pUWM471. In every case high $\beta$-galactosidase levels (about 2000 Miller units) were observed, which is consistent with previously published data that ranked the cjaA promoter as one of the the strongest Campylobacter spp. promoters so far described [39].
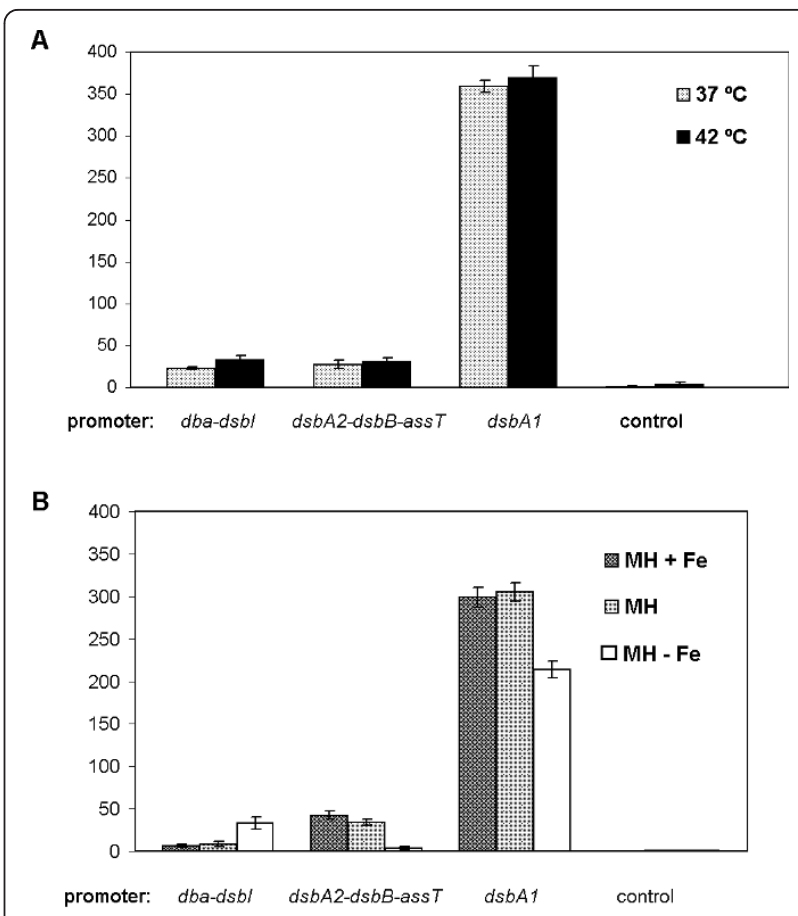

C

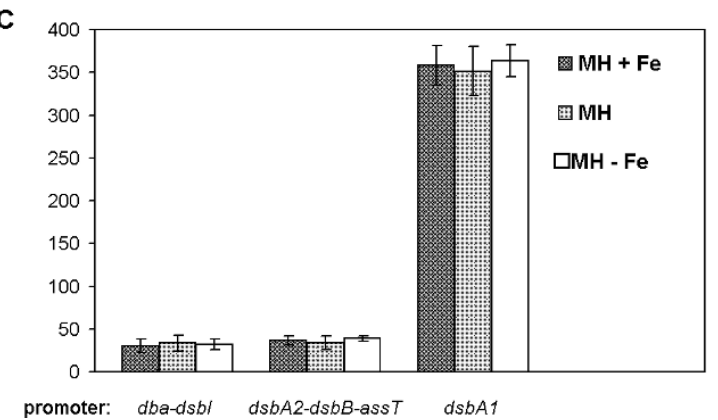

Figure 2 Transcription levels of $C$. jejuni 81-76 dsb genes (measured by $\beta$-galactosidase activity assays) in the wild type strain (A and B) and fur::cat mutant (C) under different environmental conditions. Each experiment was repeated three times, and each time three independent samples were taken for each strain (giving 9 independent measurements for each strain). Statistical significance was calculated using t-Student test for comparison of independent groups (GraphPad Prism). The wild type strain C. jejuni 480 carrying an empty vector pMW10 was used as a control. Statistical $p$ values: For wild type C. jejuni 480 strain: $P_{d b a-d s b l}$ temp. $37^{\circ} \mathrm{C}$ vs $42^{\circ} \mathrm{C}: \mathrm{p}=0,0001\left(^{*}\right)$. $\mathrm{P}_{\text {dsbaz-dsbB-astA }}$ temp. $37^{\circ} \mathrm{C}$ vs $42^{\circ} \mathrm{C}$ : $\mathrm{p}=0,2020$. $\mathrm{P}_{d s b A 1}$ temp. $37^{\circ} \mathrm{C}$ vs $42^{\circ} \mathrm{C}: \mathrm{p}=0,1031$. $\mathrm{P}_{d b a-d s b /} \mathrm{MH}+\mathrm{Fe}$ vs $\mathrm{MH}: \mathrm{p}=0,0576 . \mathrm{P}_{d b a-d s b l} \mathrm{MH}-\mathrm{Fe}$ vs $\mathrm{MH}: \mathrm{p}<0,0001\left(^{*}\right) . \mathrm{P}_{d s b A 1-d s b B-a s t A}$ $M H+F e$ vs $M H: p=0,0007\left(^{*}\right)$. $P_{d s b A 1-d s b B-a s t A} M H-F e$ vs $M H: p<$ $0,0001\left(^{*}\right) \cdot P_{d s b A 1} \mathrm{MH}+\mathrm{Fe}$ vs $\mathrm{MH}: \mathrm{p}=0,2569$. $\mathrm{P}_{\text {dsbal }} \mathrm{MH}-\mathrm{Fe}$ vs $\mathrm{MH}: \mathrm{p}<$ $0,0001\left(^{*}\right)$. For mutant C. jejuni 480 fur:: cat strain: $P_{d b a-d s b l} M H+F e$ vs

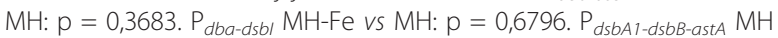
+Fe vs $M H: p=0,3164$. $P_{\text {dsbA1-dsbB-astA }} M H$-Fe vs $M H: p=0,0577$. $P_{d s b A T} M H+F e$ vs $M H: p=0,5228 . P_{d s b A 7} M H-F e$ vs $M H: p=0,2388 . P$ values of $\mathrm{P}<0.05$ were considered to be statistically significant; they are marked with $\left(^{*}\right)$. 
Inspection of the nucleotide sequences located upstream of the $d b a$ translation initiation codon did not reveal the presence of an exact $C$. jejuni Fur-binding site sequence motif [40]. So far, a potential Fur binding site for promoters positively regulated by iron concentration in a Fur-dependent manner has not been determined. Therefore, we used EMSA to gain insight into the mechanism by which $\mathrm{P}_{d b a d s b I}, \mathrm{P}_{d s b A 2 d s b B a s t A}$ and $\mathrm{P}_{d s b A 1}$ are regulated by Fur. To achieve this goal, various primers were designed to amplify a 174 - 299 bp DNA fragment upstream from the translational start site of each tested operon. The promoter region of the $c h u A$ gene, which contains the Fur-binding motif and is strongly repressed by iron-complexed Fur, was used as a control $[6,40] . \mathrm{Mn}^{2+}$ ions were used in the EMSA in place of $\mathrm{Fe}^{2+}$ due to their greater redox stability. It was demonstrated that the Fur-His ${ }_{6}$ was able to bind in vitro to the DNA region upstream of the $d b a-d s b I$ operon only when the regulatory protein was complexed with $\mathrm{Mn}^{2+}$, which indicated, in accordance with previously presented data, that this operon is repressed by the iron-complexed form of Fur (Figure 3E). This promoter region interacts with Fur complexed with $\mathrm{Mn}^{2+}$ as much as the chuA promoter (Figure 3G). In contrast, the upstream DNA region of the $d s b A 1$ gene did not bind Fur, regardless of the presence of $\mathrm{Mn}^{2+}$ in the reaction buffer. This suggested an indirect method of regulation (Figure 3, panel $\mathrm{C}$ and $\mathrm{D}$ ). In the case of the $d s b A 2-$ $d s b B$-ast $A$ promoter region, Fur protein bound DNA in the absence of $\mathrm{Mn}^{2+}$ acted as a repressor (Figure 3B), supporting the results obtained in the $\beta$-galactosidase assays. Fur-His ${ }_{6}$ complexed with $\mathrm{Mn}^{2+}$ was also able to bind to this DNA fragment but only under conditions of high protein concentration. The formation of DNA/Fur complexes specific for the $d s b A 2-d s b B$-ast $A$ promoter region was efficiently inhibited by adding unlabelled DNA containing the same DNA fragment.

To check whether the abundance/activity of Dsbdependent proteins is conditioned by iron concentration, we compared the arylsulfate sulfotransferase (AstA) activity in $C$. jejuni 81-176 wt cells grown under ironrestricted to iron-sufficient/iron-rich conditions. As mentioned before, arylsulfatase is a periplasmic direct substrate of the Dsb oxidative pathway [41-43]. This experiment confirmed the dependence of AstA activity on iron concentration. AstA activity of C. jejuni 81-176 wt grown under iron-restricted conditions reached $75-80 \%$ of activity observed for the same strain grown under iron-rich condition (Additional file 1).

\section{C. jejuni dba-dsbl translational coupling}

Previously performed in vitro transcription/translation coupled assays suggested that $C$. jejuni Dba may influence DsbI synthesis and/or stability [18]. To reveal details of $d b a-d s b I$ operon expression we examined whether $d b a / D b a$ was required for in vivo synthesis of DsbI in E. coli cells. It was demonstrated that in E. coli, DsbI underwent partial degradation (for details see Additional file 2 and 3). This result was in agreement with those derived from previous in vitro experiments. It is noteworthy that in C. jejuni cells, DsbI is produced in two forms as a result of posttranslational modification by glycan binding (for details see Additional file 2 and 4).

Additionally a C. jejuni 81-176 isogenic $d b a$ mutant was constructed by inserting the kanamycin resistance cassette in the same orientation as $d b a$ coding sequence. This insertion should not alter the downstream $d s b I$ transcription. Nevertheless, inactivation of C. jejuni dba resulted in the absence of DsbI, and subsequent RT-PCR experiments, conducted for four independently isolated transformants, also documented the absence of $d s b I$ transcript in $d b a$ mutated cells (data not shown). To further examine the role of $d b a$ expression in DsbI synthesis, a double mutant strain - C. jejuni $\Delta d b a$-dsbI::cat (AG6) was constructed. Thereafter three recombinant shuttle plasmids, pUWM769 (containing the wild type C. jejuni dba-dsbI operon), pUWM811 and pUWM812 (containing point mutated $d b a-M 1 R$ or $d b a$ : L29stop, respectively, and wild type $d s b I$ ) were introduced into mutant cells. Transformant cells were screened for DsbI synthesis by Western blot analysis with specific rabbit antirDsbI serum and additionally by RT-PCR for the presence of $d s b I$ transcript. Introduction of pUWM769 into $C$. jejuni 81-176 AG6 ( $\Delta d b a-d s b I:: c a t)$, cells resulted in restoration of DsbI production in a higher amount compared to the wild type strain (Figure 4, lane 6), due to plasmid-encoded $d b a-d s b I$ gene expression. When $d b a$ translation was completely aborted (C. jejuni AG6 carrying pUWM811) and when the truncated 28 aa Dba was produced (C. jejuni AG6/pUWM812), DsbI was not synthesized at all (Figure 4, lane 4 and 5, respectively). RT-PCR experiments proved that point mutations in $d b a$ did not influence $d s b I$ transcription, as comparable amounts of $d s b I$ mRNA were detected in all but one (AG6) of the strains (Figure 5, lanes: 9, 11-13). Comparable results were obtained for series of $C$. jejuni dsbI::cat strains carrying pUWM769, pUWM811 and pUWM812 plasmids (data not shown), suggesting that intact, chromosomally-encoded Dba cannot act in-trans to ensure $d s b I$ mRNA translation.

To further address the role of Dba in $d s b I$ expression the recombinant plasmid lacking the $d b a$ gene but containing the $d s b I$ gene transcribed from own promoter was constructed and introduced into the C. jejuni 81-176 $\Delta d b a-d s b I:: c a t$ mutant. The C. jejuni 81-176 $\Delta d b a-d s b I::$ cat, harbouring pUWM769 was employed as a control. Western experiments showed that an individual expression of the $d s b I$ gene from own promoter results in DsbI 


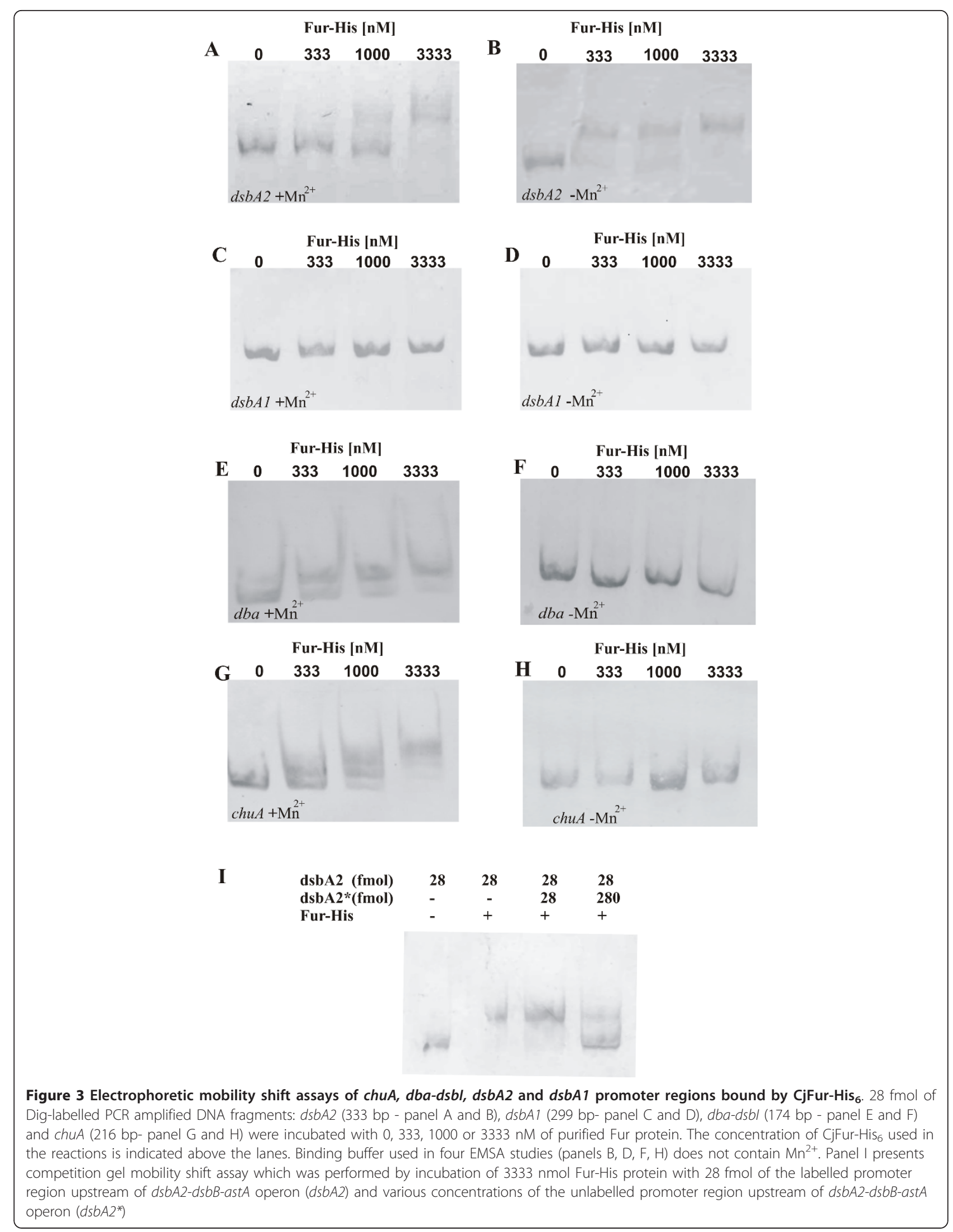




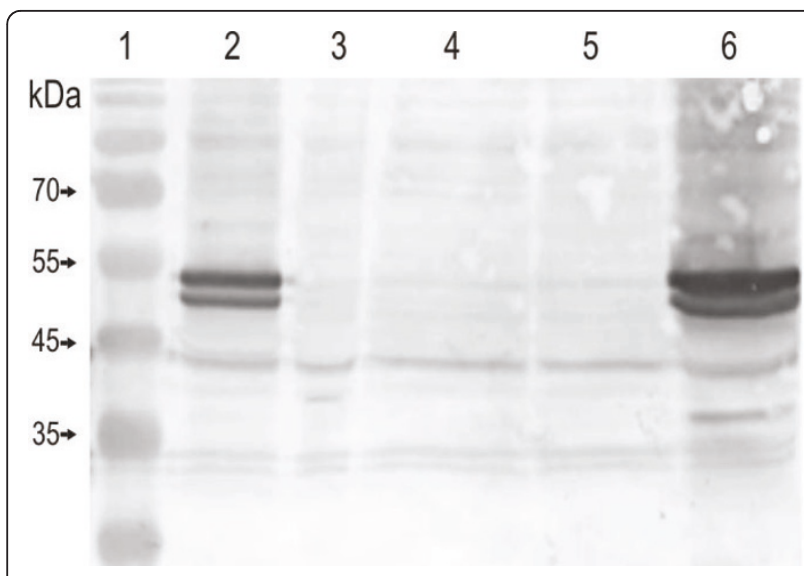

Figure 4 Translational coupling of C. jejuni dba-dsbl. Western blot (anti-rDsbl) analysis of $C$. jejuni protein extracts separated by $12 \%$ SDS-PAGE. Relative positions of molecular weight markers (lane 1) are listed on the left (in kilodaltons). Lanes 2-6 contain $15 \mu \mathrm{g}$ of total proteins from: C. jejuni 81-176 wt (2), C. jejuni 81176 AG6 (dba-dsbl::cat) (3), AG6/pUWM811 (4), AG6/pUWM812 (5) and AG6/pUWM769 (6)

production (Figure 6, lane 2), underlining once more the importance of mRNA secondary structure for the $d s b I$ mRNA translation.

\section{Discussion}

The best characterized Dsb oxidative system, that of E. coli $\mathrm{K}-12$, consists of two oxidoreductases, periplasmic DsbA and inner membrane DsbB, that are involved in disulfide bond formation de novo in the bacterial periplasm. Genes encoding these proteins are located in different chromosomal sites and are transcribed as monocistronic units.

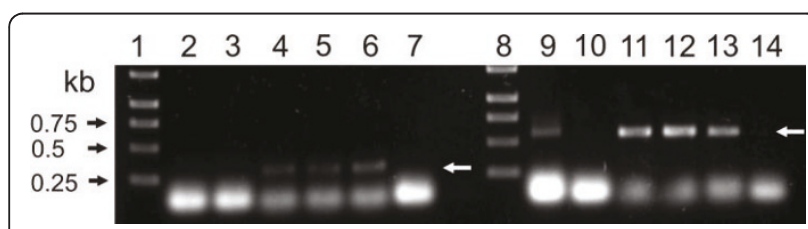

Figure 5 Analysis of $C$. jejuni $d s b /$ transcription from a $d b a-d s b l$ operon containing wild type or point mutated $d b a$. RT-PCR analysis of $d s b l$ (and aphA-3) transcription in C. jejuni wild type and mutant cells. Equal amounts of mRNAs isolated from C. jejuni cells were reverse-transcribed using primer KM-R1 or Cj-RT and resulting CDNA was PCR-amplified with primer pairs KM-L1 - KM-R1 (lanes 17) or CjNde - Cj17RM (lanes 8-14), respectively. Relative positions of DNA molecular length markers (lanes 1,8) are listed on the left (in base pairs). Lanes 2-6 and 9-13 contain PCR products amplified on cDNAs for C. jejuni 81-176 wt (2, 9), AG6 (dba-dsbl::cat) (3, 10), AG6/ pUWM811 (4, 11), AG6/pUWM812 (5, 12), AG6/pUWM769 (6, 13); lanes 7 and 14 contain PCR products amplified on RNA for AG6/ pUWM769 (after DNase treatment). White arrows indicate products of expected size.

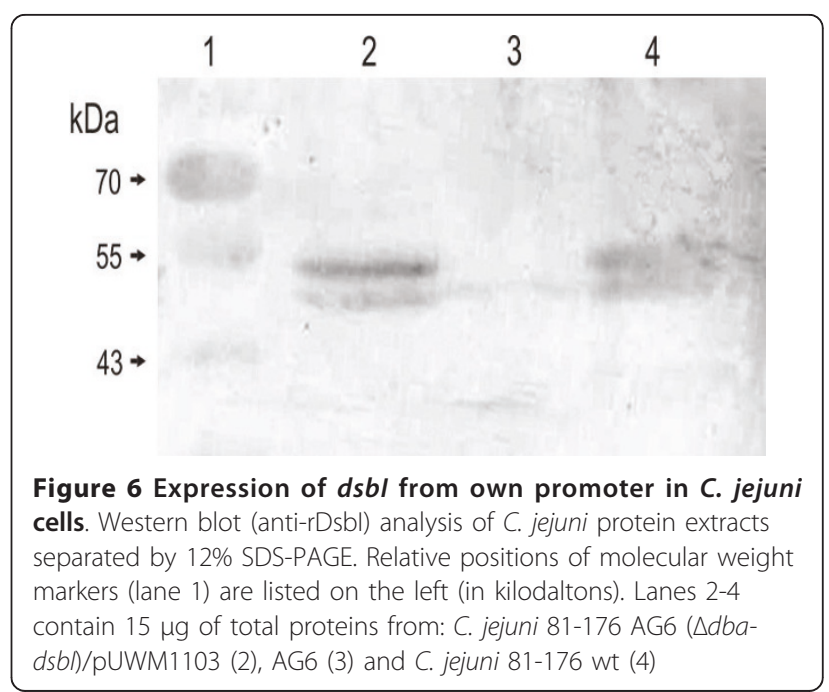

The Campylobacter jejuni Dsb oxidative pathway is more complex. In the present study we initiated analysis of $C$. jejuni $d s b$ gene organization and regulation. Our results document organization of these genes in two operons, one comprised of $d b a$ and $d s b I$, and another of $d s b A 2, d s b B$ and $a s t A$. The $d s b A 1$ gene constitutes a separate monocistronic transcriptional unit. Predictions based on in silico analysis by Petersen et al. [44] of the C. jejuni NCTC 11168 genome nucleotide sequence stated that the $d b a$ and $d s b I$ genes are cotranscribed. They also indicated that $c j 0864$ (a truncated version of $d s b A 2$ ) and $c j 0865(d s b B)$ potentially form an operon. The first $\mathrm{T}$ base of the TATA box was predicted to be located 199 bp upstream from the ATG start codon for the $d b a-d s b I$ operon and 66 bp from the ATG start codon for the $d s b A 2-d s b B$-astA operon [44].

Global comparative $C$. jejuni transcriptome or proteome analysis revealed that transcription levels of $d s b A 2, d s b B$ and $a s t A$ increase in strains isolated from a chicken cecum compared with strains grown in vitro [5] and they are down-regulated under iron-restricted conditions in vitro [6]. Stinzi et al. found that $d s b$ gene transcription was not dependent on the temperature of in vitro growth $\left(37\right.$ vs $\left.42^{\circ} \mathrm{C}\right)$ [45]. So far only one transcriptomic study has documented that $d b a$ and $d s b I$ transcript abundance is iron-dependent. Interestingly, the authors stated that the transcription of $d b a$ and $d s b I$ was antagonistically regulated by iron accessibility, depending on the experimental conditions, i. e. iron-activated shortly after iron addition into the medium and iron-repressed in the mid-log phase of growth [40]. All cited transcriptomic experiments were conducted on mRNA derived from C. jejuni NCTC 11168, a strain which has the shorter, non-functional $d s b A 2$ version.

Our experiments, conducted on C. jejuni 480 wild type expressing $\beta$-galactosidase from different $d s b$ gene 
promoters of C. jejuni 81-176, demonstrated that they are all regulated in response to iron availability. Our data are generally consistent with those derived from transcriptomic analysis. The strongest of the analyzed promoters, $\mathrm{P}_{d s b A 1}$, which was down-regulated in iron starvation conditions, was not identified in comparative transcriptomic experiments conducted by Holmes et al., although that work revealed $\mathrm{P}_{d s b A 2 d s b B a s t A}$ iron dependence [6]. Such inconsistency of experimental data might be due to limited sensitivity of the transcriptomic strategy previously used. The transcription level of $d s b A 1$ is only slightly affected by iron concentration, whereas the transcription level from $\mathrm{P}_{\text {dsba2dsbBast } A}$ decreases about 10 -fold in response to iron deficiency. The $d s b$ gene promoters are antagonistically regulated by iron availability, at least under conditions used in this study. Thus, abundance of both periplasmic oxidoreductases, DsbA1 and DsbA2, decreases when iron becomes restricted, while DsbB and DsbI membrane oxidoreductases are synthesized constitutively, in different extracellular iron concentrations. This might suggest that iron-storage proteins or non-essential iron-using proteins might be direct or indirect targets of the Dsb oxidative pathway involving activity of DsbA1/DsbB or DsbA2/DsbB redox pairs.

In some microorganisms, positive regulation by Fur and iron is provided by action of sRNAs which are themselves regulated by iron-complexed Fur - these sRNAs pair with their target mRNAs and promote their degradation (reviewed in [46]). However, $\mathrm{P}_{d s b A 2 d s b B a s t A}$ and $\mathrm{P}_{d s b A 1}$ promoters are not regulated that way, since the level of $\beta$-galactosidase in iron-sufficient medium is comparable in wild-type and fur mutated cells. This observation proved that these promoters are not induced by iron-bound Fur, as the level of $\beta$-galactosidase expressed from these two fusions is higher in response to iron limitation in the fur mutant than in the wild type cells. The most probable explanation of these results is that iron-free Fur is capable of repressing their transcription. Palyada et al. [40] performed in silico analysis aimed at Campylobacter Fur box identification. They inspected 16 DNA fragments located upstream of iron and Fur repressed genes, which allowed them to establish the potential Fur box sequence motif. However, only eleven of the analyzed promoters included this element [40]. So far C. jejuni's potential Fur box for apo-Fur repressed genes remains undetermined.

In the present study the EMSA assays confirmed that although all the analyzed promoters were members of the Fur regulon, each of them was regulated by a different mechanism. We showed that both iron-free and ironcomplexed Fur can act as a repressor. The observed potential dual regulation of the $\mathrm{P}_{d s b A 2 d s b B a s t A}$ promoter, dependent on Fur concentration, still remains unclear.
An explanation for this phenomenon requires deeper understanding of the $C$. jejuni fur gene expression. In contrast to $E$. coli, the $C$. jejuni fur gene expression is not autoregulated, and additionally, the iron-responsive Fur regulator of $C$. jejuni is expressed from two separate promoters [47]. Our findings further indicate that transcription under iron-starvation can be controlled by Fur indirectly, as was observed for the $d s b A 1$ gene. The sophisticated mechanism regulating $d s b$ gene transcription in response to iron availability may be responsible for subtle changes in the abundance and/or activity of various substrates in the Dsb system. We demonstrated that activity of $C$. jejuni 81-176 AstA, which is a direct target of Dsb system, is dependent on iron level in the medium. However, as AstA level is dependent on the activities of both DsbA1 and DsbA2 (unpublished results), details of the process remain unclear.

Recently performed comparative Helicobacter pylori and Neisseria gonorrhoeae transcriptomic analysis also indicated that genes included in the Fur regulon can be positively or negatively regulated in response to iron availability $[38,48]$. Like C. jejuni Fur, H. pylori Fur also binds to some promoters in its iron-free form to repress their expression [38,49-51]. C. jejuni Fur reveals a relatively high degree of amino acid identity with $H$. pylori Fur. Nonetheless it is not able to complement apo-Fur regulation in an $H$. pylori fur mutant when delivered in trans [52]. Such unexpected results might be due to subtle differences in conformation of both proteins. Additional experiments, such as solving the three dimensional structure of $C$. jejuni Fur, are required to clarify the functional differences between Fur proteins of these closely related species. Although both species have AT-rich genomes and some of their promoters have similar structure, it can not be excluded that the C. jejuni apo-Fur binding nucleotide sequences are not identical as those determined for $H$. pylori apo-Fur. Also two $H$. pylori promoters, the $p f r$ and sod gene promoters that are repressed by apo-Fur, exhibited low sequence similarity and revealed different affinities for apo-Fur $[38,50]$.

The second part of our research was aimed at understanding the relationship between $d b a$ and $d s b I$ expression. Experiments employing point mutated $d b a$ provided evidence for strong translational coupling of the $d b a$ and $d s b I$ genes. Inhibition or premature termination of $d b a$ mRNA translation resulted in the lack of DsbI. This defect was not complemented by the intact chromosomal dba gene in C. jejuni 81-176 dsbI::cat. Translational coupling has already been described and is common among functionally related bacterial genes. It was documented that in many cases it involves operons containing overlapping genes as well as genes constituting an operon and divided by short intergenic region 
[53,54]. C. jejuni 81-176 $d b a$ and $d s b I$ do not overlap, but are separated by a relatively short intergenic region (11 $\mathrm{bp}$ ). Experiments employing a recombinant plasmid that expressed only DsbI verified the importance of the $d b a$ $d s b I$ mRNA secondary structure for its translation. Preliminary prediction of the secondary structure for the mRNA region spanning the entire $d b a$ gene and the 5' end of the $d s b I$ gene, indicated that the $d s b I$ RBS is located within a stem-loop structure formed by a sequence fragment upstream of the RBS (including the 3' part of the $d b a$ gene) as well as one downstream of the RBS and spanning the initiator codon of the $d s b I$ gene. This suggests that mRNA translation of the $d s b I$ gene may be blocked due to the occlusion of the RBS, and that translation of the $d b a$ mRNA may make the RBS of the $d s b I$ gene accessible and hence enable the translation of the $d s b I$ gene as well. Verification of this hypothesis requires further analysis.

This coupling mechanism may facilitate interaction between two proteins expressed from the same operon. Data obtained in our study showed that in the absence of Dba, DsbI is intensively degraded in E. coli cells. Also in C. jejuni $\Delta d b a-d s b I:: c a t$ cells harboring a recombinant plasmid enabling expression of only DsbI, this protein migrates on SDS-PAGE slightly faster than DsbI produced by wild type cells. It was suggested by in silico analysis that the N-terminal domain of DsbI contains five transmembrane helixes and its $\mathrm{C}$-terminal domain achieve a $\beta$-propeller structure and localize in the periplasm [18]. DsbI localization in the inner-membrane was documented by a cell fractionation experiment (data not shown). In silico prediction also localizes Dba in the IM. Although the specific mechanism of Dba and DsbI interplay is yet unknown, we hypothesize that Dba can act as a periplasmic or transmembrane chaperone, providing the proper folding of the DsbI C-terminal domain, which might be a prerequisite for recruiting other proteins to form an active protein complex.

\section{Conclusions}

The present work documents that iron concentration is a significant factor influencing $d s b$ gene transcription. Preliminary results of proteomic experiments aimed at identification of Campylobacter Dsb system targets suggest that mutations in $d s b$ genes influence the level of a dozen extracytoplasmic proteins (manuscript in preparation). One of them is the periplasmic LivJ protein, which contains four cysteine residues and is involved in the colonization process as shown by Hendrixon and DiRita [55]. Moreover proteomic analysis of iron-regulated $C$. jejuni protein expression done by Holmes et al. showed that LivJ abundance is iron-dependent. Because livJ gene transcription is not iron nor Fur dependent, most likely the changes in the abundance of this protein are influenced by activity of the Dsb system [6]. Taken together, these results support the notion that iron concentration -through the influence on $d s b$ gene expression - might control abundance of the extracytoplasmic proteins during different stages of infection. Our work further shows that the synthesis of the DsbI membrane oxidoreductase is controlled by a translational coupling mechanism. Among bacterial genomes sequenced so far, those of C. jejuni strains are extremely compact. About $95 \%$ of their content is occupied by protein-coding regions and more than $25 \%$ of all genes overlap. Presumably, translational coupling occurs during expression of many other C. jejuni operons containing tail-to-head oriented genes with short or no intergenic regions.

\section{Additional material}

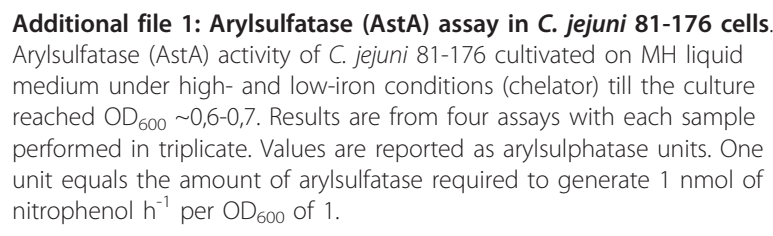

Additional file 2: Experiment details concerning Dsbl stability and glycosylation.

Additional file 3: Influence of the $d b a / D b a$ on Dsbl stability in

E. coli cells. Western blot (anti-rDsbl) analysis of C. jejuni/E. coli protein extracts separated by 12\% SDS-PAGE. Relative positions of molecular weight markers (lane 1) are listed on the left (in kilodaltons). Lanes 2-7 contain $20 \mu \mathrm{g}$ of total proteins from: C. jejuni 81-176 wt (2), E. coli/ pBluescript II KS (3), E. coli/pUWM453 (dba-dsbl) (4), E. coli/pUWM454 (dba) (5), E. coli/pUWM455 (dsbl) (6) and E. coli/pUWM456 (dba-dsbl) (7)

Additional file 4: Dsbl glycosylation. Western blot (anti-rDsbl) analysis of C. jejuni protein extracts separated by $12 \%$ SDS-PAGE. A - proteins isolated from C. jejuni 81-176 wt and pglB isogenic mutant. Relative positions of molecular weight markers (lane 1) are listed on the left (in kilodaltons). Lanes 2 and 3 contain $20 \mu \mathrm{g}$ of total proteins from: C. jejuni 81-176 wt (2) and C. jejuni 81-176 pglB:.:Cat (3). B - proteins isolated from C. jejuni 480 AL4 (dsbl::cat) overexpressing Dsbl or the mutated version of the protein Dsbl. Relative positions of molecular weight markers (lane 1) are listed on the left (in kilodaltons). Lanes 2-4 contain $20 \mu \mathrm{g}$ of total proteins from: C. jejuni 480 AL4/pUWM762 (Dsbl N292A) (2), AL4/ pUWM765 (Dsbl N340A) (3) and AL4/pUWM769 (the shuttle plasmid containing a wild type copy of the C. jejuni dsb/ gene) (4)

\section{Acknowledgements}

We thank Jeff Hansen for critical reading of the manuscript. We also thank Ewa Kosykowska for performing some complementation experiments as well as Lukasz Kozlowski and Janusz M. Bujnicki for RNA sequence analysis. This work was supported by two grants from Polish Ministry of Science and Higher Education (No. 2P04C 01527 and N N303 341835).

\section{Author details}

${ }^{1}$ Department of Bacterial Genetics, Institute of Microbiology, University of Warsaw, Miecznikowa 1, 02-096, Warsaw, Poland. ${ }^{2}$ Department of Molecular Mechanisms of Mycobacterial Infections, Institute of Pharmacology and Structural Biology, 205, route de Narbonne, 31077 Toulouse cedex, France. ${ }^{3}$ Division of Protein and Nucleic Acid Chemistry MRC Laboratory of Molecular Biology Hills Road, Cambridge, CB2 OQH, UK. ${ }^{4}$ Department of Gastroenterology, The Medical Centre of Postgraduate Education, Marymoncka 99/103, 01-813 Warsaw, Poland. 


\section{Authors' contributions}

ADG conducted out most of the laboratory work. MW and MN, working under supervision of EKJK and ADG, contributed to construction of some transcriptional fusion, mutated C. jejuni strains and translational coupling experiments. AML did RT-PCR experiments for the $d b a-d s b /$ operon as well as expression of $d s b /$ from its own promoter, and was involved in drafting the manuscript. RG performed experiments concerning influence of iron concentration on cjaA gene expression and AstA activity level. PR performed EMSA assays. AW performed experiments concerning Dsbl glycosylation. EKJK conceived the study. EKJK and ADG designed the experiments, and were engaged in data interpretation and drafting the manuscript. All authors read and accepted the final version of the manuscript.

Received: 12 April 2011 Accepted: 25 July 2011 Published: 25 July 2011

\section{References}

1. Young KT, Davis LM, Dirita VJ: Campylobacter jejuni: molecular biology and pathogenesis. Nat Rev Microbiol 2007, 5(9):665-679.

2. Moore JE, Corcoran D, Dooley JS, Fanning S, Lucey B, Matsuda M, McDowell DA, Megraud F, Millar BC, O'Mahony R, et al: Campylobacter. Vet Res 2005, 36(3):351-382.

3. Reid AN, Pandey R, Palyada K, Naikare H, Stintzi A: Identification of Campylobacter jejuni genes involved in the response to acidic $\mathrm{pH}$ and stomach transit. Appl Environ Microbiol 2008, 74(5):1583-1597.

4. Malik-Kale P, Parker CT, Konkel ME: Culture of Campylobacter jejuni with sodium deoxycholate induces virulence gene expression. J Bacteriol 2008, 190(7):2286-2297.

5. Woodall CA, Jones MA, Barrow PA, Hinds J, Marsden GL, Kelly DJ, Dorrell N, Wren BW, Maskell DJ: Campylobacter jejuni gene expression in the chick cecum: evidence for adaptation to a low-oxygen environment. Infect Immun 2005, 73(8):5278-5285.

6. Holmes K, Mulholland F, Pearson BM, Pin C, McNicholl-Kennedy J, Ketley JM, Wells JM: Campylobacter jejuni gene expression in response to iron limitation and the role of Fur. Microbiology 2005, 151(Pt 1):243-257.

7. Stintzi A, Marlow D, Palyada K, Naikare H, Panciera R, Whitworth L, Clarke C: Use of genome-wide expression profiling and mutagenesis to study the intestinal lifestyle of Campylobacter jejuni. Infect Immun 2005, 73(3):1797-1810

8. Sampathkumar B, Napper S, Carrillo CD, Willson P, Taboada E, Nash JH, Potter AA, Babiuk LA, Allan BJ: Transcriptional and translational expression patterns associated with immobilized growth of Campylobacter jejuni. Microbiology 2006, 152(Pt 2):567-577.

9. Kalmokoff M, Lanthier P, Tremblay TL, Foss M, Lau PC, Sanders G, Austin J, Kelly J, Szymanski CM: Proteomic analysis of Campylobacter jejuni 11168 biofilms reveals a role for the motility complex in biofilm formation. J Bacteriol 2006, 188(12):4312-4320

10. Wosten MM, Parker CT, van Mourik A, Guilhabert MR, van Dijk L, van Putten JP: The Campylobacter jejuni PhosS/PhosR operon represents a non-classical phosphate-sensitive two-component system. Mol Microbiol 2006, 62(1):278-291.

11. Raphael BH, Pereira S, Flom GA, Zhang Q, Ketley JM, Konkel ME: The Campylobacter jejuni response regulator, CbrR, modulates sodium deoxycholate resistance and chicken colonization. J Bacteriol 2005, 187(11):3662-3670

12. Bras AM, Chatterjee S, Wren BW, Newell DG, Ketley JM: A novel Campylobacter jejuni two-component regulatory system important for temperature-dependent growth and colonization. J Bacteriol 1999, 181(10):3298-3302

13. Wosten MM, Wagenaar JA, van Putten JP: The FlgS/FlgR two-component signal transduction system regulates the fla regulon in Campylobacter jejuni. J Biol Chem 2004, 279(16):16214-16222.

14. Mackichan JK, Gaynor EC, Chang C, Cawthraw S, Newell DG, Miller JF, Falkow S: The Campylobacter jejuni dccRS two-component system is required for optimal in vivo colonization but is dispensable for in vitro growth. Mol Microbiol 2004, 54(5):1269-1286.

15. Lasica AM, Jagusztyn-Krynicka EK: The role of Dsb proteins of Gramnegative bacteria in the process of pathogenesis. FEMS Microbiol Rev 2007, 31(5):626-636.

16. Heras B, Shouldice SR, Totsika M, Scanlon MJ, Schembri MA, Martin JL DSB proteins and bacterial pathogenicity. Nat Rev Microbiol 2009, 7(3):215-225.
17. Dutton RJ, Boyd D, Berkmen M, Beckwith J: Bacterial species exhibit diversity in their mechanisms and capacity for protein disulfide bond formation. Proc Natl Acad Sci 2008, 105(33):11933-11938.

18. Raczko AM, Bujnicki JM, Pawlowski M, Godlewska R, Lewandowska M, Jagusztyn-Krynicka EK: Characterization of new DsbB-like thioloxidoreductases of Campylobacter jejuni and Helicobacter pylori and classification of the DsbB family based on phylogenomic, structural and functional criteria. Microbiology 2005, 151(1):219-231.

19. Yao R, Guerry P: Molecular cloning and site-specific mutagenesis of a gene involved in arylsulfatase production in Campylobacter jejuni. $J$ Bacteriol 1996, 178(11):3335-3338.

20. Kwon AR, Choi EC: Role of disulfide bond of arylsulfate sulfotransferase in the catalytic activity. Arch Pharm Res 2005, 28(5):561-565.

21. Malojcic G, Owen RL, Grimshaw JP, Brozzo MS, Dreher-Teo H, Glockshuber R: A structural and biochemical basis for PAPS-independent sulfuryl transfer by aryl sulfotransferase from uropathogenic Escherichia coli. Proc Natl Acad Sci 2008, 105(49):19217-19222.

22. Lasica AM, Wyszynska A, Szymanek K, Majewski P, Jagusztyn-Krynicka EK: Campylobacter protein oxidation influences epithelial cell invasion or intracellular survival as well as intestinal tract colonization in chickens. J App/ Genet 2010, 51(3):383-393.

23. Korlath JA, Osterholm MT, Judy LA, Forfang JC, Robinson RA: A pointsource outbreak of campylobacteriosis associated with consumption of raw milk. J Infect Dis 1985, 152(3):592-596.

24. Wassenaar TM, Fry BN, van der Zeijst BA: Genetic manipulation of Campylobacter: evaluation of natural transformation and electrotransformation. Gene 1993, 132(1):131-135.

25. van Vliet AH, Wooldridge KG, Ketley JM: Iron-responsive gene regulation in a Campylobacter jejuni fur mutant. J Bacteriol 1998, 180(20):5291-5298.

26. Sambrook J, Russel DW: Molecular cloning: a laboratory manual. Cold Spring Harbor New York: Cold Spring Harbor Laboratory Press; 2001.

27. Yao R, Alm RA, Trust TJ, Guerry P: Construction of new Campylobacter cloning vectors and a new mutational cat cassette. Gene 1993, 130(1):127-130.

28. Ditta G, Stanfield S, Corbin D, Helinski DR: Broad host range DNA cloning system for gram-negative bacteria: construction of a gene bank of Rhizobium meliloti. Proc Natl Acad Sci 1980, 77(12):7347-7351.

29. Labigne-Roussel A, Harel J, Tompkins L: Gene transfer from Escherichia coli to Campylobacter species: development of shuttle vectors for genetic analysis of Campylobacter jejuni. J Bacteriol 1987, 169(11):5320-5323.

30. Davis L, Young K, DiRita V: Genetic manipulation of Campylobacter jejuni. Curr Prot Microbiol 2008, Chapter 8, Unit 8A 2 1-8A 217.

31. Miller JH: Experiments in molecular genetics. Cold Spring Harbor Laboratory Press New York: Cold Spring Harbor; 1972.

32. Wright JA, Grant AJ, Hurd D, Harrison M, Guccione EJ, Kelly DJ, Maskell DJ: Metabolite and transcriptome analysis of Campylobacter jejuni in vitro growth reveals a stationary-phase physiological switch. Microbiology 2009, 155(Pt 1):80-94.

33. Hendrixson DR, DiRita VJ: Transcription of sigma54-dependent but not sigma28-dependent flagellar genes in Campylobacter jejuni is associated with formation of the flagellar secretory apparatus. Mol Microbiol 2003, 50(2):687-702.

34. Wosten MM, Boeve M, Koot MG, van Nuenen AC, van der Zeijst BA: Identification of Campylobacter jejuni promoter sequences. J Bacteriol 1998, 180(3):594-599.

35. Delany I, Grifantini R, Bartolini E, Rappuoli R, Scarlato V: Effect of Neisseria meningitidis fur mutations on global control of gene transcription. J Bacteriol 2006, 188(7):2483-2492.

36. Lee HW, Choe YH, Kim DK, Jung SY, Lee NG: Proteomic analysis of a ferric uptake regulator mutant of Helicobacter pylori: regulation of Helicobacter pylori gene expression by ferric uptake regulator and iron. Proteomics 2004, 4(7):2014-2027.

37. Delany I, Rappuoli R, Scarlato V: Fur functions as an activator and as a repressor of putative virulence genes in Neisseria meningitidis. Mol Microbiol 2004, 52(4):1081-1090.

38. Ernst FD, Bereswill S, Waidner B, Stoof J, Mader U, Kusters JG, Kuipers EJ, Kist M, van Vliet AH, Homuth G: Transcriptional profiling of Helicobacter pylori Fur- and iron-regulated gene expression. Microbiology 2005, 151(Pt 2):533-546. 
39. Wyszynska A, Pawlowski M, Bujnicki J, Pawelec D, Van Putten JP, Brzuszkiewicz E, Jagusztyn-Krynicka EK: Genetic characterisation of the cjaAB operon of Campylobacter coli. Pol J Microbiol 2006, 55(2):85-94.

40. Palyada K, Threadgill D, Stintzi A: Iron acquisition and regulation in Campylobacter jejuni. J Bacteriol 2004, 186(14):4714-4729.

41. Totsika M, Heras B, Wurpel DJ, Schembri MA: Characterization of two homologous disulfide bond systems involved in virulence factor biogenesis in uropathogenic Escherichia coli CFT073. J Bacteriol 2009, 191(12):3901-3908.

42. Lin D, Kim B, Slauch JM: DsbL and Dsbl contribute to periplasmic disulfide bond formation in Salmonella enterica serovar Typhimurium. Microbiology 2009, 155(Pt 12):4014-4024.

43. Grimshaw JP, Stirnimann CU, Brozzo MS, Malojcic G, Grutter MG, Capitani G, Glockshuber R: DsbL and Dsbl form a specific dithiol oxidase system for periplasmic arylsulfate sulfotransferase in uropathogenic Escherichia coli. J Mol Biol 2008, 380(4):667-680

44. Petersen L, Larsen TS, Ussery DW, On SL, Krogh A: RpoD promoters in Campylobacter jejuni exhibit a strong periodic signal instead of a -35 box. J Mol Biol 2003, 326(5):1361-1372.

45. Stintzi A: Gene expression profile of Campylobacter jejuni in response to growth temperature variation. J Bacteriol 2003, 185(6):2009-2016.

46. Masse E, Salvail H, Desnoyers G, Arguin M: Small RNAs controlling iron metabolism. Curr Opin Microbiol 2007, 10(2):140-145.

47. van Vliet AH, Rock JD, Madeleine LN, Ketley JM: The iron-responsive regulator Fur of Campylobacter jejuni is expressed from two separate promoters. FEMS Microbiol Lett 2000, 188(2):115-118.

48. Jackson LA, Ducey TF, Day MW, Zaitshik JB, Orvis J, Dyer DW: Transcriptional and functional analysis of the Neisseria gonorrhoeae Fur regulon. J Bacteriol 2010, 192(1):77-85.

49. Danielli A, Amore G, Scarlato V: Built shallow to maintain homeostasis and persistent infection: insight into the transcriptional regulatory network of the gastric human pathogen Helicobacter pylori. PLoS Pathog 2010, 6(6):e1000938.

50. Delany I, Spohn G, Rappuoli R, Scarlato V: The Fur repressor controls transcription of iron-activated and -repressed genes in Helicobacter pylori. Mol Microbiol 2001, 42(5):1297-1309.

51. Danielli A, Scarlato V: Regulatory circuits in Helicobacter pylori : network motifs and regulators involved in metal-dependent responses. FEMS Microbiol Rev 2010, 34(5):738-752.

52. Miles S, Carpenter BM, Gancz H, Merrell DS: Helicobacter pylori apo-Fur regulation appears unconserved across species. J Microbiol 2010, 48(3):378-386.

53. Mathiesen G, Huehne K, Kroeckel L, Axelsson L, Eijsink VG: Characterization of a new bacteriocin operon in sakacin P-producing Lactobacillus sakei, showing strong translational coupling between the bacteriocin and immunity genes. Appl Environ Microbiol 2005, 71(7):3565-3574.

54. Waldo RH, Krause DC: Synthesis, stability, and function of cytadhesin P1 and accessory protein B/C complex of Mycoplasma pneumoniae. J Bacteriol 2006, 188(2):569-575

55. Hendrixson DR, DiRita VJ: Identification of Campylobacter jejuni genes involved in commensal colonization of the chick gastrointestinal tract. Mol Microbiol 2004, 52(2):471-484

56. Simon R, Priefer U, Puhler A: A broad host range mobilization system for in vivo genetic engineering: Transposon mutagenesis in gram negative bacteria. Nat Biotech 1983, 1(9):784-791.

doi:10.1186/1471-2180-11-166

Cite this article as: Grabowska et al:: Campylobacter jejuni dsb gene expression is regulated by iron in a Fur-dependent manner and by a translational coupling mechanism. BMC Microbiology 2011 11:166.

\section{Submit your next manuscript to BioMed Central and take full advantage of:}

- Convenient online submission

- Thorough peer review

- No space constraints or color figure charges

- Immediate publication on acceptance

- Inclusion in PubMed, CAS, Scopus and Google Scholar

- Research which is freely available for redistribution

Submit your manuscript at www.biomedcentral.com/submit
Biomed Central 